\title{
Colloque du DHURDV Kolloquium des DHURDV
}

\section{(b) NOVARTIS}

\section{Quelques principes de la chirurgie' \\ Nowartis Pharma Schweiz AG \\ CH-3001 Bern, Postiach}

\author{
A. Skaria ${ }^{a}$, D. Salomon ${ }^{b}$, R.P. Braun ${ }^{b}$ \\ ${ }^{a}$ Consultant à la Clinique de Dermatologie de Genève, Vevey, et \\ ${ }^{\mathrm{b}}$ Clinique de Dermatologie, Genève, Suisse
}

La fermeture correcte d'un défaut est essentiel pour le résultat esthétique. Ceci est d'autant plus important pour le dermatologue pour qui le soin de la peau est une carte de visite. Pour obtenir un bon résultat il faut respecter deux points importants. Il faut:

1) éviter les sutures transcutanées si possible; si on est obligé de les utiliser, il faut toujours d'abord mettre des fils souscutanés et il faut enlever les fils transcutanés tôt, c'est-à-dire au visage après au maximum 5 jours, au corps après au maximum 10 jours;

2) ne jamais fermer la plaie sous trop de tension car la tension risque de marquer les cicatrices soit par des déhiscences cicatricielles, soit par des cicatrices hypertrophiques; pour obtenir cela, il faut parfois faire des lambeaux cutanés qui permettent de redistribuer les forces de traction.

Il nous semble essentiel que des reconstructions par lambeaux devraient seulement être effectuées si on est sûr que toute la tumeur est excisée. Ceci nous ne semble pas le cas dans les excisions des carcinomes basocellulaires ou spinocellulaires du visage avec une histologie conventionnelle. Dans ces cas, nous insistons sur la performance de la chirurgie micrographique («Mohs surgery»). Les lambeaux que nous montrons sont destinés à des excisions au scalp, tronc et aux extrémités après excision d'un grand nævus ou la reprise d'un mélanome malin (MM).

Fig. 1. La suture sous-cutanée profonde sert à adapter le tissu d'une excision profonde comme p.ex. la reprise d'un MM. Il est mieux de placer le nœud en profondeur, mais ce n'est pas essentiel comme dans la suture sous-cutanée superficielle. Elle peut être complétée par des sutures sous-cutanées superficielles ou transcutanées.

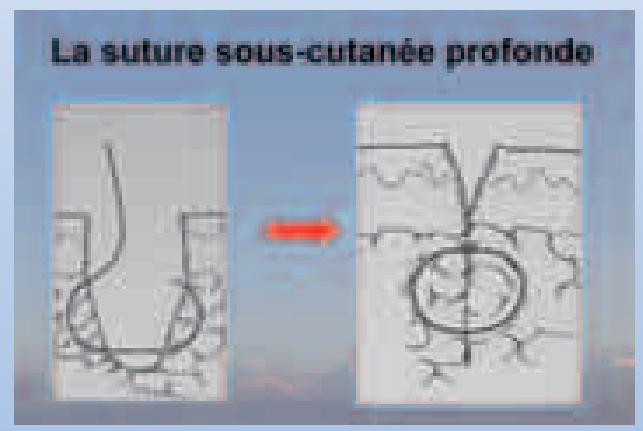
${ }^{1}$ Compte rendu réalisé avec le soutien de la firme Novartis Pharma,
Berne, Suisse.
Fig. 2. La suture sous-cutanée superficielle permet d'adapter des excisions qui vont jusqu'à l'hypoderme superficiel. Il est important que le nœud se trouve en profondeur pour une meilleure résorption et pour éviter un granulome sur fil.

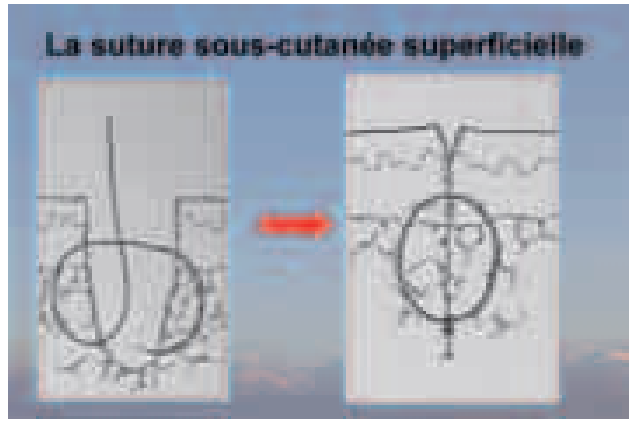

Fig. 3, 4. Les défauts de plus de $1 \mathrm{~cm}$ de longueur sont mieux fermés avec une suture sous-cutanée continue. On commence avec un point sous-cutané simple avec le nœud vers la profondeur, ensuite on continue avec le fil en commençant du derme profond et en sortant au-dessous de la jonction épidermique et vice versa jusqu'à l'autre bout de la cicatrice.
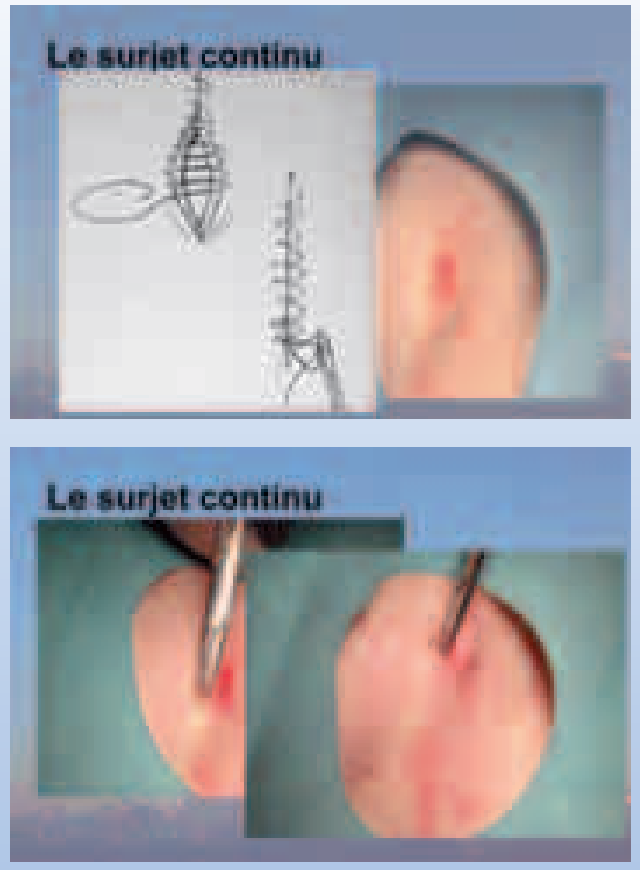
Fig. 5-8. Le dernier point profond est gardé un peu plus long et utilisé comme une terminaison libre d'un point simple. On effectue un point trois fois comme dans une suture habituelle. A travers l'anse qui se forme il faut enfiler l'aiguille et faire un nœud. Ce nœud sécurise le dernier point et permet de cacher celui-ci dans l'hypoderme profond pour éviter de nouveau un granulome sur fil [Skaria AM: Dermatol Surg 2002;28:739-741].
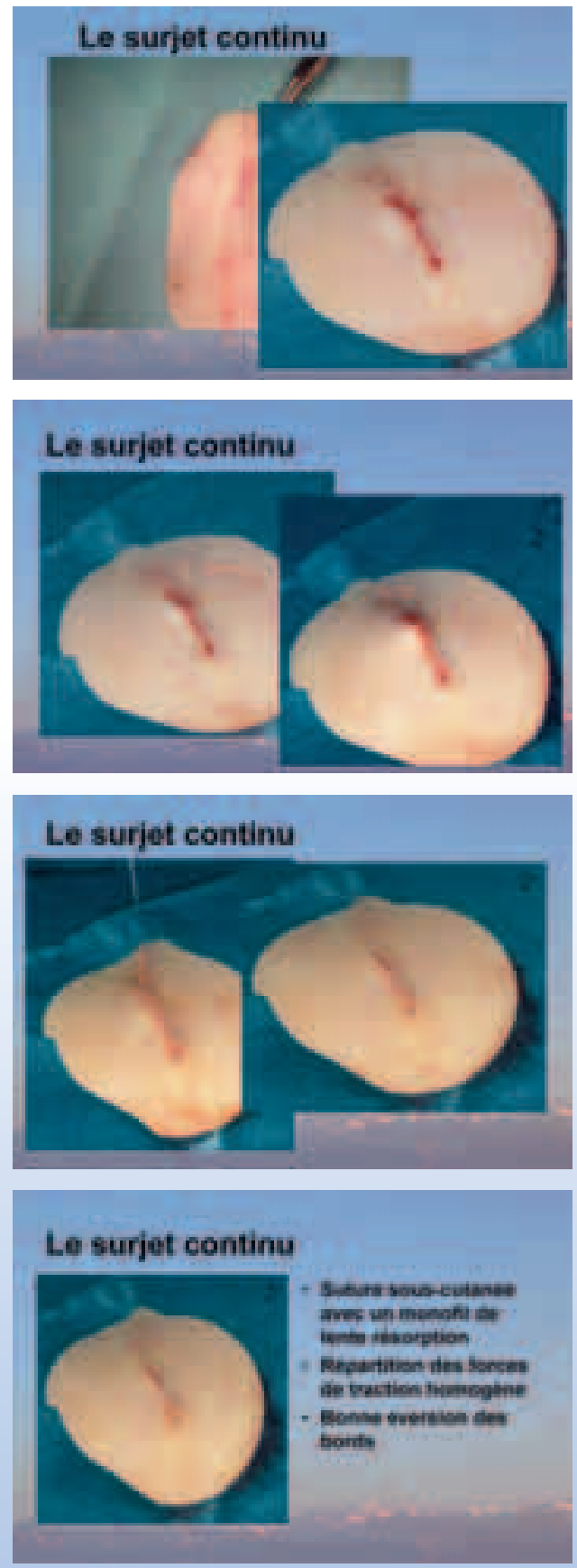

Fig. 9. La suture «Donati modifié» est une suture très utile pour des petites excisions. On commence par un point profond du derme à l'épiderme. On ré-entre dans le derme par le point où on est sorti. On glisse l'aiguille sous l'épiderme pour sortir entre la jonction dermo-épidermique.

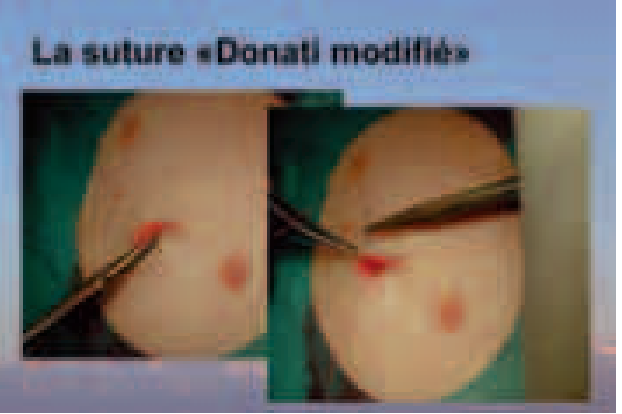

Fig. 10, 11. On entre sur l'autre berge de la plaie à la hauteur de la jonction dermo-épidermique et on sort vers l'épiderme. On ré-entre l'aiguille vers la même ouverture où on est sorti et on sort l'aiguille dans le derme pour effectuer les nœuds comme dans une suture sous-cutanée classique [Saurat J-H, et al: Dermatologie, 4e éd. Paris, Masson, 2004].

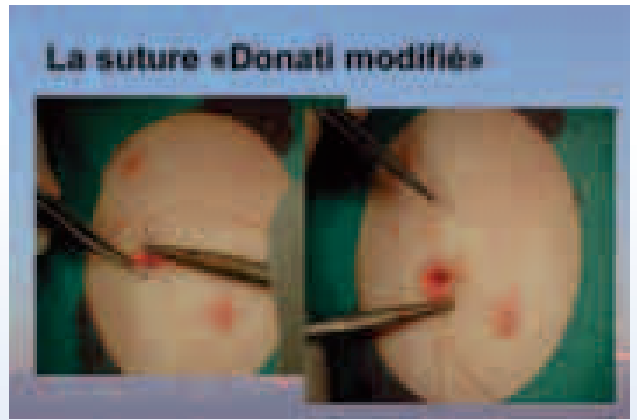

\section{La suture *Donati modifies}

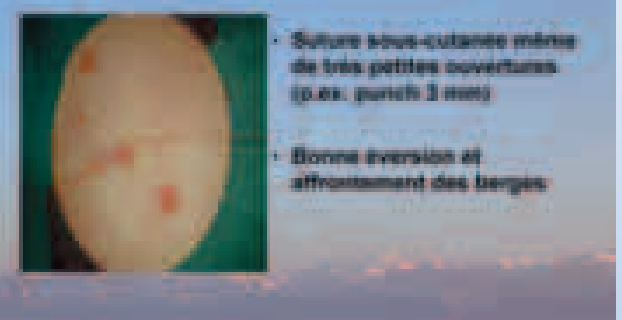


Fig. 12. Les lambeaux de rotation permettent de fermer de multiples défauts différents. Il n'y a pas seulement un lambeau de rotation, mais différentes combinaisons des lambeaux de rotation comme le lambeau de $\mathrm{O}-\mathrm{Z}$ ou les lambeaux à rotations multiples. Ces lambeaux sont surtout utiles au scalp, tronc et aux extrémités.

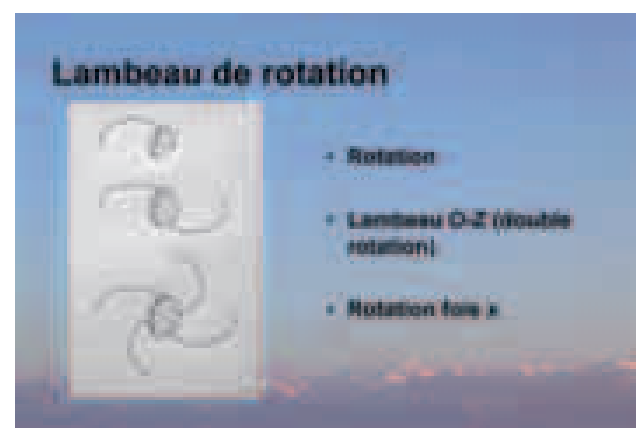

Fig. 13. Cette patiente de 29 ans a subi de multiples excisions d'un chirurgien pour un carcinome basocellulaire du scalp fermées ensuite par une greffe de peau totale. Cette sorte de fermeture est inadéquate dans cette localisation chez une jeune patiente.

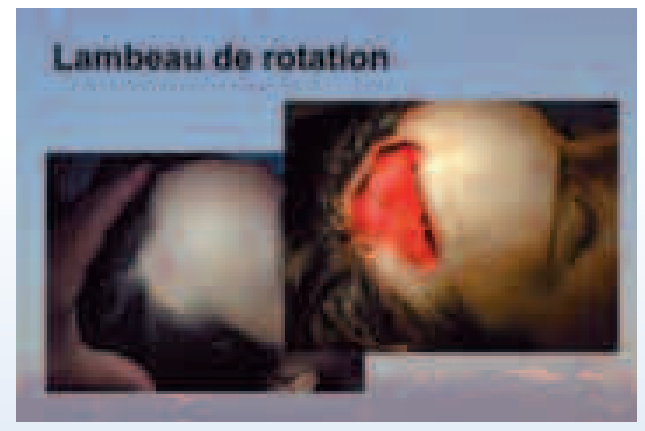

Fig. 14. Si une fois on est sûr que toute la tumeur a été enlevée, une fermeture par un lambeau est indiquée. La meilleure option dans cette localisation est un lambeau de rotation pour recréer au mieux la lisière du cuir chevelu.

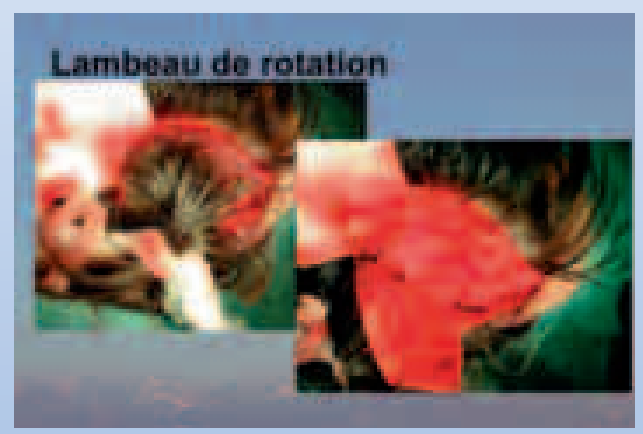

\section{Colloque du DHURDV \\ Kolloquium des DHURDV}

Fig. 15. Dans la planification d'un lambeau du cuir chevelu, il faut faire attention à la direction de l'insertion du poil pour maintenir si possible celle-ci. Si on effectue la rotation, on va créer un petit «dog-ear»; ceci est important car il peut servir comme petite greffe de peau totale si nécessaire ou comme réservoir pour des lambeaux à rotations multiples.

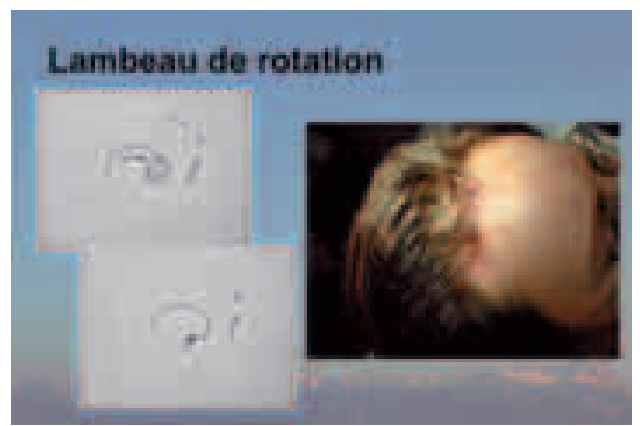

Fig. 16. Dans certaines situations, un lambeau de rotation simple n'est pas suffisant raison pour laquelle il faut modifier et adapter la technique. Chez ce patient de 40 ans avec une reprise d'un MM avec une marge de $2 \mathrm{~cm}$ (1998 avant que la dissection du nœud lymphatique sentinelle ne s'effectuât aux scalps), le défaut ne se fut pas fermé avec un lambeau de rotation simple.

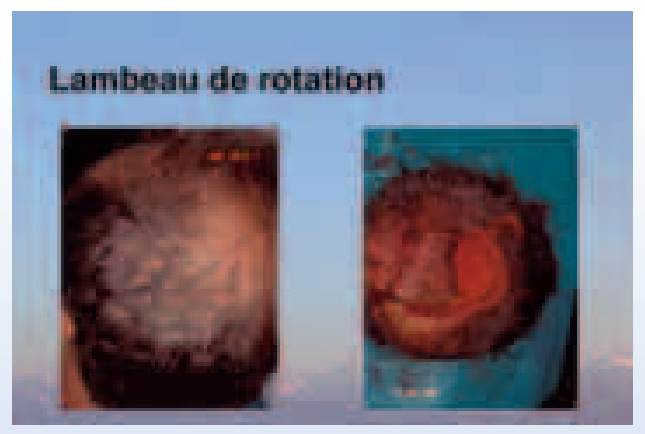

Fig. 17. On peut toujours dessiner un premier lambeau de rotation et si la fermeture est trop sous tension on peut procéder à un deuxième lambeau de rotation en contre-sens. Ceci fait que ce lambeau est nommé O-Z.

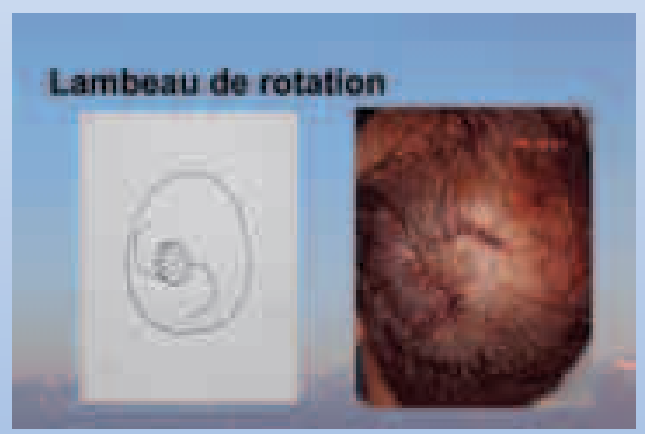




\section{Colloque du DHURDV \\ Kolloquium des DHURDV}

Fig. 18, 19. Si le défaut est encore plus grand comme chez cette patiente de 86 ans avec un MM de $4 \mathrm{~cm}$ de diamètre avec en plus une marge de $2 \mathrm{~cm}$, multiples lambeaux de rotation sont nécessaires.
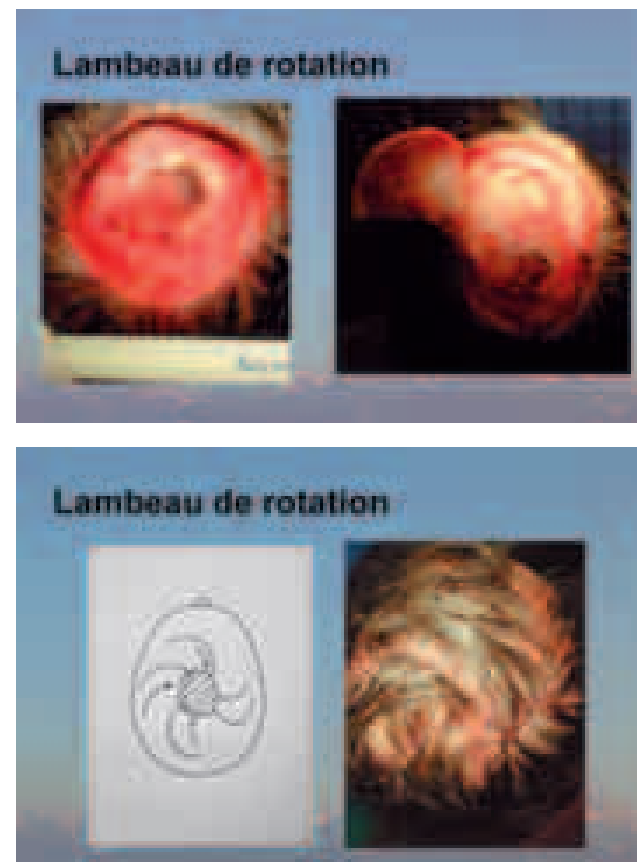

Fig. 20, 21. Le chirurgien qui a voulu faire la fermeture de ce défaut a fait multiples erreurs. 1) Il a dessiné un lambeau A-T en voulant faire une rotation. 2) Le réservoir de peau dans un lambeau A-T est minime car il s'agit d'un lambeau d'avancement qui n'est pas adapté dans cette localisation. 3) Le réservoir de la rotation est beaucoup plus grand et permet plus de mobilisation de tissu. 4) Malgré une grande tension sur les berges, le chirurgien a fermé la plaie en prenant le risque d'avoir des nécroses. 5) Une fermeture par greffe de Burrows aurait été préférable comparée à la solution démontrée. 6) La solution idéale aurait été un lambeau O-Z comme dessiné à droite.

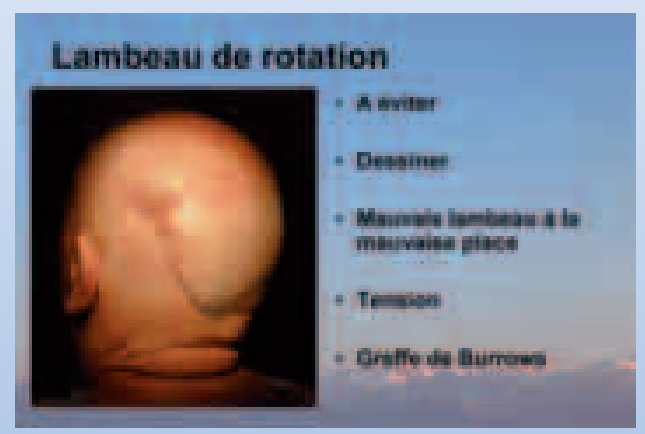




\section{Colloque du DHURDV \\ Kolloquium des DHURDV}

Fig. 24. Remarquez la facilité avec laquelle on peut mobiliser ce lambeau et le résultat après 5 jours. Pas de discoloration ischémique, pas de nécrose. Le lambeau en ilôt avec des modifications décrit est idéal pour les reconstructions sur les extrémités et le tronc. L'avantage de ce lambeau est de nouveau la possibilité de le combiner avec un deuxième lambeau. Ce lambeau a aussi fait preuve dans les lésions péri-nasales et des joues. Dans ces localisations, il faut bien connaître l'anatomie et le drainage lymphatique pour ne pas susciter des problèmes car le pédicule est enraciné profondément.

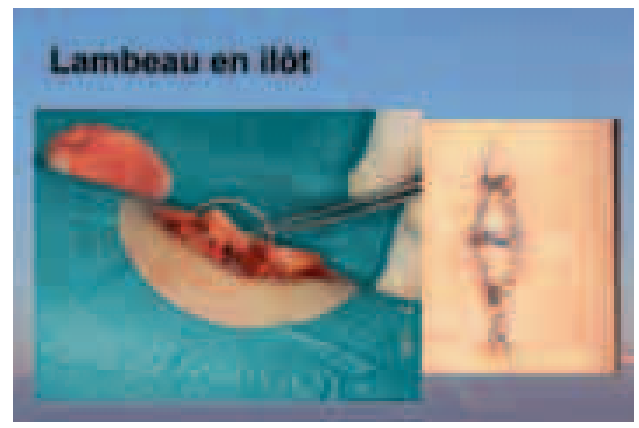

\section{Département hospitalo-universitaire romand de dermatologie et vénéréologie - Dates des colloques du jeudi en 2005}

Attention: Avant de vous déplacer, veuillez recontrôler ces dates sur la feuille des colloques que vous recevez chaque mois.

Avril 2005

Jeudi, 7 avril Colloque de formation continue + journal-club à Genève

Jeudi, 28 avril Colloque DHURDV à Nyon organisé par Lausanne

Mai 2005

Jeudi, 12 mai Colloque de formation continue + journal-club à Genève

Jeudi, 26 mai Colloque DHURDV à Nyon organisé par Genève

Juin 2005

Jeudi, 9 juin Colloque de formation continue + journal-club à Genève

Jeudi, 30 juin Colloque DHURDV à Nyon organisé conjointement par Genève et Lausanne

\section{Revolutionary and amazing}

\section{Rong Xiang Xu}

\section{Burns}

\section{Regenerative Medicine and Therapy}

\author{
Editor: Xia Sun \\ Co-Editor: Bradford S. Weeks
}

Collaboration of Mo Xiao - Xiangqing Zhang • Junxiang Zhao • Chengqun Luo • Zenglu Xu • Ruiqing Zhao • Guangshun Wang • Hongsheng Wang - Doncai Hu

'Regenerative Medicine' is an innovative concept representing a unique approach to the regeneration of functional tissues and organs. This book reveals the scientific principles behind this newly discovered practice while instructing the reader in the procedure of MoistExposed Burns Therapy (MEBT) and offering compelling examples of tissue and organ regeneration from ordinary cells incubated in potent nutrient baths.

This publication will add a new dimension to the discussions on burns treatment, stem cells, immunology and cell biology. Burns specialists will learn of the new gold standard in burns treatment, and cell biologists of the potential of ordinary cells.

\section{Contents \\ Preface}

Brief Introduction to the History of Burns Medical Science Introduction

Rationale Foci of Local Treatment of Burns Medicine and Therapy Evaluation and Classification of Burn Severity

Clinical Principles of Burns Regenerative Medicine and Therapy Experimental and Clinical Study on Burns Regenerative Medicine and Therapy with MEBT/MEBO

Clinical Demonstrations of Burns Regenerative Medicine and Therapy (MEBT/MEBO) on Successful Treatment of Extensive Burns

Clinical Results of Surgical Excision and Skin Grafting Therapy in the Treatment of Extensive Burns Patients

A Commentary on Burns Medical and Regenerative Therapy Conclusion

For the full contents please see our website: www.karger.com/burns

Xu, R.X. (Beijing)

Burns Regenerative Medicine and Therapy

Editors: Sun, X. (Beijing); Weeks, B.S. (Clinton, Wash.)

VIII + 152 p., 69 fig., 39 in color, 68 tab., hard cover, 2004

CHF 227.50 / EUR 162.50 / USD 198.00

Prices subject to change

EUR price for Germany, USD price for USA only ISBN 3-8055-7661-7 